\title{
Re-evaluation of the left atrial to aortic root ratio as a marker of patent ductus arteriosus
}

\author{
Parvathi Iyer, Nick Evans
}

\begin{abstract}
The aim of this study was to re-examine the accuracy of the left atrial aortic root ratio (LA:Ao) as a marker of significant patent ductus arteriosus (PDA) in the preterm infant by comparison with direct Doppler echocardiographic assessment. Fifty six infants $(<1500 \mathrm{~g})$ had 463 serial echocardiograms. Firstly the LA:Ao was measured, then the duct was imaged and classified as wide open, restricting, or closed according to two dimensional and Doppler criteria. Probability analysis was performed to test the ability of the LA:Ao to discriminate between a wide open PDA and a restricting or closed duct.

Mean LA:Ao was $1 \cdot 17$ and $1 \cdot 21$ when the duct was respectively closed or restricting compared with 1.61 when wide open. Using a LA:Ao of 1.5 as a cut off gives a sensitivity of $79 \%$ and specificity of $95 \%$ and increases the accuracy over the recommended levels of 1.3 and $1 \cdot 4$. With this cut off there were 20/94 false negatives, these were associated with scans on day 1 and large interatrial shunts. The sensitivity of the LA:Ao increased to $88 \%$ if only scans performed after day 1 were analysed. For diagnosing a PDA after day 1, the positive likelihood ratio of an LA:Ao of 1.5 or more was $17 \cdot 5$, and the negative likelihood ratio of an $\mathrm{LA}$ :Ao $<1 \cdot 5$ was $0 \cdot 13$.

The LA:Ao is still a useful tool in the diagnosis of PDA. It is a simple method which needs less skill and resources than direct PDA imaging and is feasible on neonatal units without direct access to echocardiographic expertise. Its use on the first postnatal day is not recommended.
\end{abstract}

(Arch Dis Child 1994; 70: F112-F117)

Department of Perinatal Medicine, King George V Hospital for Mothers and Babies, Part of Royal Prince Alfred Hospital, Sydney, New South Wales, Australia Parvathi Iyer

Nick Evans

Correspondence to:

Dr Nick Evans, Department of Perinatal Medicine, King George V Hospital for Mothers and Babies, Missenden Road,

Camperdown, Sydney, NSW 2050, Australia.

Accepted 28 September

1993
Persisting patency of the ductus arteriosus is an important complication of neonatal intensive care. The National Collaborative Study reported an incidence of symptomatic patent ductus arteriosus (PDA) varying from $11 \%$ to $36 \%$ in the 13 different units, with a higher incidence in infants less than 30 weeks gestation with respiratory distress. ${ }^{1}$

Clinical diagnosis of a haemodynamically significant PDA lacks accuracy, particularly in the early postnatal days. ${ }^{2-4}$ Both false negative and false positive clinical findings are common in the first postnatal week. ${ }^{5}$ Clinical pointers such as increased arterial pulse pressure have been shown not to differ in preterms with PDA. ${ }^{6}$ As a result, echocardiography has become an important non-invasive method for confirming ductal patency. Historically, the first marker of ductal patency was the $M$ mode measurement of the ratio of the left atrial diameter to that of the aortic root (LA:Ao). ${ }^{78}$ The LA:Ao depends on the fact that left to right ductal shunting increases the volume load on the left side of the heart so the left atrium will dilate relative to the aortic root which will be relatively unaffected by the volume load. Published normal mean values for LA:Ao and that which supposedly indicates the presence of a PDA vary widely. ${ }^{8-10} \mathrm{But}$, in the recent literature, the levels of LA:Ao most commonly taken to indicate the presence of a significant PDA are above $1 \cdot 3^{3}$ and $1 \cdot 4 .^{10}$ The LA:Ao as a diagnostic tool for PDA fell into disfavour as a result of three studies that questioned its accuracy. Hirschklau et al described seven of 31 infants with signs of a PDA and a normal LA:Ao. ${ }^{9}$ Valdez-Cruz et al found, using contrast echocardiography as a gold standard, that the left atrial diameter was inaccurate in preterm infants who were fluid restricted and ventilated, the misclassification rate of LA:Ao being $51 \% .{ }^{11}$ And Kupferschmid et al found, with clinical signs as a gold standard, a sensitivity of only $50 \%$ using a value of $1 \cdot 4: 1$ as the cut off for LA:Ao. ${ }^{4}$ The problem with these studies are the 'gold standards' used for evaluating the accuracy of the LA:Ao. Clinical or radiographic signs have been shown to be inaccurate in the diagnosis of a significant $\mathrm{PDA}^{2-5}$ and contrast echocardiography will detect all ductal shunts and will only loosely differentiate those of haemodynamic significance.

The most accurate echocardiographic method for diagnosing PDA is direct imaging with colour flow and pulsed wave Doppler analysis of the shunt. ${ }^{12-15}$ These techniques allow assessment of the internal diameter of the PDA ${ }^{16}$ and the direction of the shunt. A comparison of the LA:Ao with these direct imaging methods has not, to our knowledge, been previously performed. Direct two dimensional and Doppler echocardiographic assessment of the ductus arteriosus needs considerable echocardiographic expertise and training and sophisticated ultrasound technology. Many neonatal units do not have direct access to such skills. In contrast, LA:Ao measurement is a very easy technique to learn, requires only basic ultrasound technology, and is thus more universally applicable in newborn intensive care areas. With this in mind the aim of this study was to re-examine the accuracy of the LA:Ao using the gold standard of direct ductal imaging with two dimensional 
echocardiography with pulsed and colour flow Doppler.

\section{Methods}

Fifty six preterm infants weighing less than $1500 \mathrm{~g}$ with respiratory distress needing more than 12 hours of artificial ventilation were studied. Their mean gestation was 27 weeks (range 24-31 weeks) and the mean birth weight $965 \mathrm{~g}$ (range 512-1500). Eighteen babies were studied at the John Radcliffe Hospital, Oxford between February and August 1991. Thirty eight neonates were studied at King George V Hospital, Sydney between February and December 1992. In Oxford, echocardiography was done with an Ultramark 4 scanner using a $7 \cdot 5 \mathrm{MHz}$ probe which incorporated a $5 \mathrm{MHz}$ pulsed Doppler crystal. In Sydney, the infants were studied with an Accuson 128XP/10 scanner using a $7.5 \mathrm{MHz}$ probe incorporating both pulsed and colour flow Doppler. Serial echocardiographic studies were performed daily from day 1 to day 7, twice in the second week of life, and when possible on day 28 , as well as before discharge or transfer, whenever the babies stayed long enough in the unit.

In Oxford, the procedure for each study was as follows: firstly, $M$ mode pictures of the left atrium and the aortic root were obtained from a parasternal long axis view. The left atrial and the aortic root diameters were measured, using the incorporated measurement calipers, by the 'leading edge' technique based on the recommendations of the American Society of Echocardiography. ${ }^{17}$ The aortic root diameter was measured from the anterior edge of the anterior wall to the inner or anterior boundary of the posterior aortic wall, at the end of ventricular diastole. The left atrial dimensions were measured at the end of ventricular systole, from the anterior boundary of the posterior aortic wall to the subendocardial surface of the posterior left atrial wall. A mean of three cycles were used to compute the LA:Ao. Secondly, the ductus was imaged along the length of its course from a high right parasternal window, the pulsed Doppler gate was placed in the pulmonary end of the duct and the flow pattern from the shunt recorded. The ductus was classified into the following three groups. A 'wide open' ductus was one which was clearly patent throughout its course, with a strong easily recorded pulsed Doppler signal from the ductal shunt. A 'restricting' ductus was one which showed a definite constriction within the duct course, with weak pulsed Doppler signals from the shunt. A 'closed' ductus was one which was not patent, with no detectable shunt on a pulsed Doppler search of the pulmonary end of the duct. The flow pattern of any ductal shunt was classified as left to right, bidirectional, or right to left. In the first two patterns, the peak velocity of the left to right component of the shunt was measured and, in the bidirectional shunts, the time of right to left shunt relative to the right ventricular ejection time was measured.

In Sydney, the echocardiographic procedure was similar but with the following differences.
The echocardiogram was recorded onto videotape and the measurement taken at a later date. The LA:Ao was averaged from a mean of three cardiac cycles. The use of colour flow mapping was incorporated into the classification of the direct imaging of the duct. In a wide open duct the colour flow mapping within the duct had a diameter of more than or equal to $1.5 \mathrm{~mm}$, in a restricting duct it was less than $1.5 \mathrm{~mm}$ and a closed duct had no detectable shunt on colour flow mapping. In addition, in these 38 infants a pulsed Doppler recording was made of the pattern of flow in the postductal descending aorta. The direction of flow in diastole was classified as antegrade, no flow or retrograde. Finally, left ventricular ejection fraction was measured from a parasternal $M$ mode recording, using a standard method. ${ }^{17}$

The clinicians who made decisions regarding treatment were blind to the echocardiographic findings unless there were signs or symptoms of a PDA when the findings would be made available to them. The decision to treat an infant was made by the attending clinicians, using both clinical and echocardiographic information. The study protocol was approved by both hospital ethical committees and the infants were studied with the consent of their parents.

\section{STATISTICS}

Sensitivity, specificity, and positive and negative predictive values were computed for the LA:Ao to determine which level best predicted significant patency of a ductus as demonstrated on direct two dimensional Doppler echocardiography. Predictive values are unstable and are affected by the prevalence rates of a condition in different populations. Likelihood ratios were also calculated as they have the advantage of being independent of prevalence rates and are powerful markers of a test's accuracy. ${ }^{18} 19$ The positive or abnormal likelihood ratio (LR) is the increase in post-test probability, if the test is abnormal, that a PDA is present relative to the pretest probability. The pretest probability being the chance that a PDA is present before any test has been done, that is the prevalence of PDA. It is calculated by the formula:

$$
\text { Positive } L R=\frac{\text { true positives }}{\text { total with condition }} \times \frac{\text { total without condition }}{\text { false positives }}
$$

The negative or normal likelihood ratio is the decrease in post-test probability, if the test is normal, that a PDA is present compared with the pretest probability. It is worked out by the formula:

$$
\text { Negative } L R=\frac{\text { false negatives }}{\text { total with condition }} \times \frac{\text { total without condition }}{\text { true negatives }}
$$

The higher the positive likelihood ratio and the lower the negative likelihood ratio, the more accurate a test is. The probability analysis is 
expressed with 95\% confidence intervals (CI). ${ }^{19}$ The one tailed $t$ test was used as a test of significance, with $p$ values of $<0.05$ being considered significant.

\section{Results}

A total of 463 echocardiograms were performed on 56 babies. On 283 occasions the duct was closed. The duct was patent on 180 occasions. Of these, in 94 instances the duct was classified as wide open and on 86 occasions the ductus was classified as restricting. The mean (SD) LA:Ao, when the ductus was closed was $1 \cdot 17(0 \cdot 2)$. In those where the duct was restricting, the mean LA:Ao was $1 \cdot 21$ $(0.2)$; this difference was just significant, $\mathrm{p}<0.05$. When the ductus was wide open, the mean LA:Ao was $1.61(0.27)$, this was significantly greater than for closed or restricting ducts, either analysed seperately or combined, $\mathrm{p}<0.0001$.

DESCENDING AORTIC DOPPLER FLOW PATTERNS In the 38 infants in whom it was assessed, the descending aortic diastolic flow was retrograde or absent in $94 \%$ of occasions when the duct was wide open compared with $9 \%$ with absent diastolic aortic flow when the duct was restricting. No infant showed retrograde diastolic flow in the restricting group.

\section{DUCTAL SHUNT DOPPLER ASSESSMENT}

On the 94 occasions the PDA was felt to be wide open the ductal flow pattern was left to right in $74(79 \%)$, bidirectional in $19(20 \%)$, and right to left in one (1\%). The mean (SD) peak velocity of the left to right component of the shunt was $1.37(0.56) \mathrm{m} / \mathrm{sec}, \mathrm{n}=85$ (this data was not collected in nine studies). This increased from $1.03(0.32) \mathrm{m} / \mathrm{sec}$ on day 1 to $1.24(0.35) \mathrm{m} / \mathrm{sec}$ on day 2 and to $1.55(0.62)$ $\mathrm{m} / \mathrm{sec}$ after this time. Bidirectional shunting was seen on only three occasions after day 2 ; the mean time of right to left shunting relative to the right ventricular ejection time was $37 \%$ $(17 \%), n=18$. Thus, where the shunt was bidirectional, this was left to right for a mean of $63 \%$ of systole and throughout diastole.

In the 86 restricting ducts the flow pattern was left to right in $82(95.5 \%)$ and bidirectional in four $(4.5 \%)$. The mean peak velocity of the left to right component of the shunt was $1.75(0.65) \mathrm{m} / \mathrm{sec}, \mathrm{n}=77$ (this data was not collected in nine studies). The mean velocity was $1.42(0.39) \mathrm{m} / \mathrm{sec}$ on day $1,1.42(0.54)$ $\mathrm{m} / \mathrm{sec}$ on day 2 and to $1.92(0.66) \mathrm{m} / \mathrm{sec}$ after

Sensitivity, specificity, and positive and negative predictive values (95\% CI) for different levels of $L A: A o$ in diagnosing significant $P D A$

\begin{tabular}{lllll}
\hline LA:Ao & Sensitivity & Specificity & $\begin{array}{l}\text { Positive } \\
\text { predictive } \\
\text { value }\end{array}$ & $\begin{array}{l}\text { Negative } \\
\text { predictive } \\
\text { value }\end{array}$ \\
\hline 1.3 & $88(85$ to 95$)$ & $72(68$ to 77$)$ & $45(33$ to 55$)$ & $96(94$ to 98$)$ \\
1.4 & $82(74$ to 90$)$ & $87(83$ to 90$)$ & $61(51$ to 71$)$ & $95(93$ to 97$)$ \\
1.5 & $79(71$ to 87$)$ & $95(93$ to 97$)$ & $80(72$ to 89$)$ & $95(92$ to 97$)$ \\
1.5 (excluding day 1$)$ & $88(81$ to 95$)$ & $95(93$ to 97$)$ & $79(70$ to 88$)$ & $97(95$ to 99$)$ \\
1.6 & $51(41$ to 61$)$ & $98(97$ to 100$)$ & $87(81$ to 94$)$ & 89 (86 to 92) \\
\hline
\end{tabular}

this time. Bidirectional shunting was seen on only one occasion after day 2; the mean time of right to left shunting relative to the right ventricular ejection time was $59 \%(15 \%)$.

\section{PROBABILITY ANALYSIS}

As the difference in mean LA:Ao between the closed and restricting duct was small, probability analysis was performed to test the ability of LA:Ao as an isolated measure to discriminate between a wide open duct (that is, likely to be haemodynamically significant) and a closed or restricting duct (that is, likely to haemodynamically insignificant).

This probability analysis was performed for different levels of LA:Ao (table). A cut off of $1 \cdot 3$ as used by Mellander et al ${ }^{3}$ provides reasonable sensitivity and negative predictive value but specificity and positive predictive values of only $72 \%$ and $45 \%$. At this cut off level the positive likelihood ratio was 3.2 (95\% CI: 2.7 to 3.8 ) and negative likelihood ratio $0 \cdot 16(95 \% \mathrm{CI}$ : 0.09 to 0.28 ) (figure). Using 1.4 as a cut off for LA:Ao as recommended by Johnson et al, ${ }^{10}$ the specificity and positive predictive value increase to $87 \%$ and $61 \%$ and the positive and negative likelihood ratios to $6 \cdot 2(95 \% \mathrm{CI}: 4 \cdot 7$ to $8 \cdot 1)$ and 0.21 ( $95 \%$ CI: 0.14 to 0.32 ). The accuracy of the LA:Ao is further improved by increasing the cut off to 1.5 . The specificity and positive predictive value increase to $95 \%$ and $80 \%$ respectively without a major decrease in sensitivity or negative predictive values. The improvement in accuracy is best demonstrated by the large increase in positive likelihood ratio to $16 \cdot 1(95 \% \mathrm{CI}: 10 \cdot 2$ to $25 \cdot 6)$ with only a small increase in negative likelihood ratio to 0.22 (95\% CI: 0.15 to 0.33 ) (figure). Further increasing the cut off to 1.6 results in a large fall in sensitivity and increase in negative likelihood ratio. This data would suggest that the most accurate level of LA:Ao to discriminate between a haemodynamically significant and insignificant PDA is 1.5 .

\section{FALSE NEGATIVES}

Using a value of 1.5 as a cut off for LA:Ao, there were 20 false negatives where the LA:Ao was less than 1.5 with a wide open ductus. Twelve of these 20 studies were done in the first 24 hours of life, in one of these cases the ductal shunt was pure right to left. Further analysing these studies on day 1 , there was an insignificant trend to those with an LA:Ao of less than 1.5 having a lower maximum velocity to the left to right shunt $0.92 \mathrm{~m} / \mathrm{sec} v 1.07 \mathrm{~m} / \mathrm{sec}$ and when the shunt was bidirectional, having a longer right to left component $58 \% v 42 \%$. Recalculating the likelihood ratios for a cut off of 1.5 but excluding all scans done on day 1 results in a small increase in positive likelihood ratio to 17.5 (95\% CI: 10.9 to 28.05 ) but quite a reduction in negative likelihood ratio to $0 \cdot 13$ ( $95 \%$ CI: 0.07 to 0.24 ) (figure). Of the remaining eight false negative studies, one had a LA:Ao just below the cut off at 1.45 and one infant was preterminal when the scan was performed. We observed anecdotally unusually 


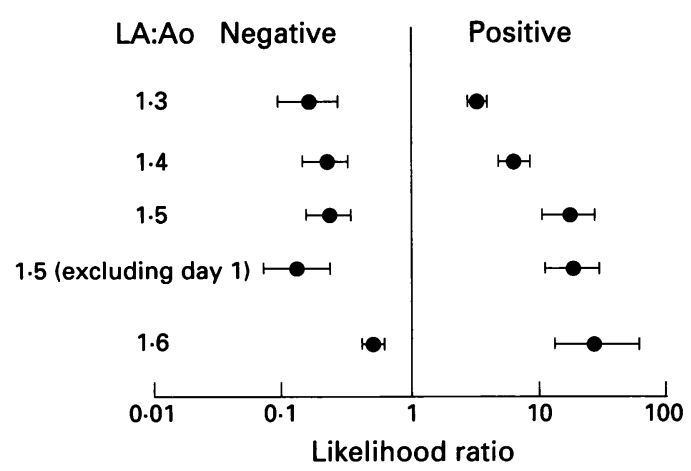

Positive and negative likelihood ratios (with $95 \%$ CI) at different levels of $L A: A o ; 1 \cdot 5$ excluding day 1 is an analysis of the likelihood ratios for a level of $L A:$ Ao of $1 \cdot 5$ excluding all scans on the first postnatal day.

large left to right atrial shunts on colour flow mapping in five of the remaining six false negatives. In none of these instances was the infant fluid restricted on the day a false negative was recorded.

\section{FALSE POSITIVES}

There were $18 / 369$ false positives where the LA:Ao was greater than 1.5 in the presence of a closed or restricting duct on direct imaging. These 18 instances occurred in 11 babies with a predominant clustering in four infants, suggesting that sometimes LA:Ao may be a reflection of individual variation in atrial shape. One of these 18 studies was in an infant with a ventricular septal defect with significant left to right shunting. There were no differences in left ventricular ejection fraction when these 18 studies were compared with true negatives, that is those when the LA:Ao was $<1.5$ in the absence of significant ductal patency. Likewise, the day of study in these 18 babies did not conform to a particular pattern.

\section{INFANTS TREATED WITH INDOMETHACIN}

Twenty one of these infants were treated for a symptomatic PDA. Treatment was directed by the development of physical signs and symptoms of a PDA rather than on the basis of the prospective echocardiography of this study. The mean (SD) LA:Ao on the day treatment was commenced, was $1 \cdot 7(0 \cdot 18)$. Two infants had a LA:Ao of less than 1.5 at the time of treatment. One of these was treated on day 1 for persisting hypotension, the preceding scan had shown a wide open PDA with a right to left shunt. In the other infant symptoms which were later shown to be septic in origin, were ascribed to a PDA - which was felt to be small on the preceding direct ductal assessment.

\section{Discussion}

This study shows that the LA:Ao is still a useful echocardiographic measurement in the diagnosis of a haemodynamically significant PDA and that previous reports which questioned its accuracy may have been exaggerated. 4911 The main problem which has dogged much of this research into the ductus arteriosus is the lack of a good non-invasive gold standard. Two of the studies that have questioned the accuracy of the LA:Ao relied on the assessment of characteristic signs and symptoms. ${ }^{49}$ These physical signs are inaccurate in early postnatal life ${ }^{2-5}$ and symptoms of a PDA can be difficult to separate from the natural fluctuations in respiratory status which occur with acute and chronic neonatal lung disease. In the other study, Valdez-Cruz et al used contrast echocardiography, a method which only provides a rough assessment of the size of the ductal shunt. ${ }^{11}$ Direct ductal assessment using two dimensional and pulsed and colour Doppler techniques is clearly now the most accurate method for diagnosis of a PDA. ${ }^{12-15}$ What is less clear is which echocardiographic measure provides the best indication of the size of the ductal shunt. ${ }^{6}$ The size of a shunt through any defect in the heart will depend on the size of the defect and the pressure differential across that defect. The primary prerequisite for a large shunt is the size of the defect, for example, the large pressure differential across a small muscular ventricular septal defect does not lead to a significant shunt. For this reason, in this study we have used, as the primary criteria of the significance of a duct, an assessment of the internal diameter of the PDA, that is, the physical size of the defect. ${ }^{16}$ We used this together with a Doppler assessment of the direction of the ductal shunt. In all except one study, the predominant direction of shunting was left to right. Where there was bidirectional shunting, the right to left component occupied a minority of the cardiac cycle. The maximum velocities of the left to right shunt showed that on the whole the aortopulmonary pressure difference fall into quite a narrow range in preterm infants with wide open ducts. The difference in the mean left to right shunt velocity on day 1 and day 3 onwards would represent an average increase in aortopulmonary pressure differences of $5.4 \mathrm{~mm} \mathrm{Hg}$. We also assessed the strength of the Doppler signal from the shunt. This is a qualitative assessment that will broadly relate to both the volume and the diameter of the jet of blood shunting through the duct. In other words, a large shunt jet will produce strong signals that are easy to record while the fine shunt jet from a restricting duct will produce weaker signals which move in and out of the pulse Doppler range gate with respiratory and cardiac movements and are thus more difficult to record. In 38 of the infants, we backed this up with a further method of assessing for significant ductal shunting, the presence of absent or retrograde diastolic flow in the descending aorta. ${ }^{20}$ This observation correlated closely with our assessment of ductal size and Doppler signal strength. Clearly, like any non-invasive technique, this is not a perfect gold standard. It is likely that there was a small degree of overlap between our wide open and restricting groups and this may have explained some of the false negatives and positives. However, we feel that this direct imaging assessment is a significant improvement on the gold standards previously used. 
If the skill and technology is available, these direct Doppler imaging methods are the best way to assess a preterm infant for a PDA. The reality is that these are not easy ultrasound methods to learn, considerable training and practice in Doppler echocardiography is needed. As a result of this many newborn intensive care units do not have immediate access to these skills. In contrast, measurement of LA:Ao is a simple technique to learn and is possible with even the most basic ultrasound machines and is thus within the scope of most neonatal units. This study has shown that the isolated measurement of the LA:Ao is a useful and reasonably accurate test for a haemodynamically significant PDA. The data has shown that increasing the cut off to 1.5 considerably increases the accuracy of the test over the previously recommended levels of $1 \cdot 4^{10}$ and $1 \cdot 3 .^{3}$ A LA:Ao of more than 1.5 was associated with positive likelihood ratio of $16 \cdot 1$, or in other words the infant would be 16 times more likely to have a significant PDA than before the test was done. And if the LA:Ao was less than $1 \cdot 5$, the negative likelihood ratio 0.22 or the infant would be five times less likely to have a significant PDA.

The problem of false negatives was the main concern raised by the studies which questioned the accuracy of the LA:Ao. ${ }^{4911}$ These studies suggested that fluid restriction or lateral rather than anteroposterior expansion of the left atrium might explain this. ${ }^{911} \mathrm{We}$ could find no evidence to support either of these hypotheses. None of these infants was fluid restricted at the time the false negative study was performed. No evidence of lateral expansion of the left atrium was seen. We did observe anecdotally, on colour flow mapping, that a high proportion of these infants had unusually large left to right interatrial shunts leading to a hypothesis that the left atrium may be decompressing into the right atrium in these cases. The most important factor in the false negatives was early postnatal scans. Twelve of the 20 false negatives were performed on the first postnatal day. One of these had a pure right to left shunt so it is not surprising that the left atrium had not dilated. In the others, the lower shunt peak velocities and higher proportions of bidirectional shunting would suggest that the left to right shunt was not sufficiently large at this time to cause the left atrium to dilate. The LA:Ao is not an accurate test on the first postnatal day. Excluding scans performed on day 1 further improved the negative likelihood ratio. If the LA:Ao was less than 1.5 after day 1 , the negative likelihood ratio was $0 \cdot 13$, or the infant would be almost eight times less likely to have a significant PDA. Although uncommon after day 1 , false negatives do occur and this needs to be taken into account when interpreting the result of this test.

In the literature, false positives have been associated with either congenital heart disease producing left to right shunting or with impaired left ventricular function. ${ }^{21}$ These data showed a relatively low incidence of false positives, $18 / 369$. One of these had a ventricular septal defect with significant left to right shunting but there was no evidence of impaired left ventricular function in these 18 studies. These 18 studies were performed in 11 babies with a predominant clustering in four of them, suggesting an individual anatomic variation which perhaps tends to falsely increase the LA:Ao.

Nineteen out of 21 infants who needed treatment for a symptomatic duct had an LA:Ao of greater than 1.5 at the time of treatment. Of the other two infants, one of these was treated on day 1 for persistent hypotension, the preceding scan had shown a large duct with a predominantly right to left shunt. The other baby had been felt to have a small PDA on echocardiographic assessment. Apnoea then developed which was put down to the PDA. However, PDA closure made no difference to the apnoea. Blood cultures later confirmed the problem was caused by septicaemia. This case highlights the problems of ascribing symptoms to a PDA.

When would the use of this test be recommended? Clearly if a unit has the facilities for direct ductal Doppler echocardiographic assessment together with other indirect measures of shunt size, this will provide optimal information. Many units do not have access to these skills and so rely on clinical pointers and physical signs to diagnose and treat PDAs. While we would not recommend use of the LA:Ao on day 1, its measurement after this time would provide useful additional information when there is a clinical suspicion of a PDA. Its use could also allow earlier diagnosis, for example in an infant without physical signs who is proving difficult to wean from ventilation.

In summary, while not a perfect test, the LA:Ao is still a useful tool in the diagnosis of a significant PDA in preterm infants. Its use would allow timely and appropriate treatment to be instituted. The accuracy of the LA:Ao is increased by using a value of 1.5 as the cut off. Its use is not recommended on the first postnatal day. It is a simple method which needs less skill and resources than direct PDA imaging and is feasible in neonatal units without direct access to echocardiographic expertise.

1 Ellison RC, Peckham GJ, Lang P, et al. Evaluation of the preterm infant for patent ductus arteriosus. Pediatrics 1983; 71: 364-72.

2 Dudell GG, Gersony WM. Patent ductus arteriosus in neonates with severe respiratory disease. $\mathcal{F}$ Pediatr 1984; 104: 915-20.

3 Mellander M, Larsson LE, Ekstrom-Jodal B, Sabel KG. Prediction of symptomatic patent ductus arteriosus in
preterm infants using Doppler and $M$-mode echocardiopreterm infants using Doppler and M-mode
graphy. Acta Paediatr Scand 1987; 76: 553-9.

4 Kupferschmid Ch, Lang D, Pohlandt F. Sensitivity, specificity and predictive value of clinical findings, m-mode ficity and predictive value of clinical findings, m-mode
echocardiography and continuous wave Doppler sonoechocardiography and continuous wave Doppler sono-
graphy in the diagnosis of symptomatic patent ductus graphy in the diagnosis of symptomatic patent ductus
arteriosus in preterm infants. Eur $₹$ Pediatr 1988; 147: 279-82.

5 Skelton R, Evans NJ, Smythe J. A blinded comparison of clinical and echocardiographic evaluation of the preterm infant for patent ductus arteriosus. F Paediatr Child Health 1993; 29: A61.

6 Evans NJ, Iyer P. Change in blood pressure after treatment of patent ductus arteriosus with indomethacin. Arch Dis Child 1993; 68: 584-7.

7 Evans NJ. Diagnosis of patent ductus arteriosus in the preterm newborn. Arch Dis Child 1993; 68: 58-61.

8 Silverman NH, Lewis AB, Heymann MA, Rudolph AM. Echocardiographic assessment of ductus arteriosus shunt in premature infants. Circulation 1974; 50: 821-5. 
9 Hirschklau MJ, DiSessa TG, Higgins CB, Friedman WF. Echocardiographic diagnosis: pitfalls in the premature infant with a large patent ductus arteriosus. $\mathcal{f}$ Pediatr 1978; 92: 474-7.

10 Johnson GL, Breart GL, Gewitz MH, et al. Echocardiographic characteristics of premature infants with patent ductus arteriosus. Pediatrics 1983; 72: 864-71.

11 Valdes-Cruz LM, Dudell GG. Specificity and accuracy of echocardiographic and clinical criteria for diagnosis of patent ductus arteriosus in fluid-restricted infants. $\mathcal{f}$ patent ductus arteriosus

12 Vick GW, Huhta JC, Gutgesell HP. Assessment of the ductus arteriosus in preterm infants utilising suprasternal two dimensional/Doppler echocardiography. $\tilde{f} \mathrm{Am} \mathrm{Coll}$ Cardiol 1985; 5: 973-7.

13 Huhta JC, Cohen M, Gutgesell P. Patency of the ductus arteriosus in normal neonates: two dimensional echocardiography vs Doppler assessment. Pediatr Cardiol 1984; 4: 561-4.

14 Reller MD, Zeigler ML, Rice MJ, Solin RC, McDonald RW. Duration of ductal shunting in healthy preterm infants: an echocardiographic color flow Doppler study. $\mathcal{f}$ Pediatr 1988; 112: 441-6.

15 Negme RA, O'Connor TZ, Lister G, Bracken MB. Patent ductus arteriosus. In: Sinclair JC, Bracken MB, eds.
Effective care of the newborn. Oxford: Oxford University Press, 1992: 281-324.

16 Ramsey JM, Murphy DJ, Vick W, et al. Response of the patent ductus arteriosus to indomethacin treatment. $A m \mathcal{F}$ Dis Child 1987; 141: 294-7.

17 Sahns DJ, DeMaria A, Kisslo J, Weyman A. Recommendations regarding quantitation in $\mathbf{M}$-mode echocardiography: results of a survey of echocardiographic measurements. Circulation 1978; 58: 107283 .

18 Mohide P, Grant A. Evaluating diagnosis and screening during pregnancy and childbirth. In: Chalmers I, Enkin M, Keirse MJNC, eds. Effective care in pregnancy and childbirth. Oxford: Oxford University Press, 1989: 66-80.

19 Simel DL, Samsa GP, Matchar DB. Likelihood ratios with confidence: sample size estimation for diagnostic test studies. $\mathcal{F}$ Clin Epidemiol 1991; 44: 763-70.

20 Serwer GA, Armstrong BE, Anderson PAW. Continuous wave Doppler ultrasographic quantitation of patent ductus arteriosus flow. F Pediatr 1982; 100: 297-9.

21 Sahn DJ, Vaucher Y, Williams DE, Allen HD, Goldberg SI, Friedman WF. Echocardiographic detection of large left to right shunts and cardiomyopathies in infants and to right shunts and cardiomyopathies
children. Am $\mathcal{F}$ Cardiol 1976; 38: 73-9. 